\title{
Daya Bay neutrino oscillation results
}

\author{
V. Vorobel *i \\ Faculty of Mathematics and Physics \\ Charles University \\ E-mail: vit.vorobel@mff.cuni.cz
}

The Daya Bay Reactor Neutrino Experiment was designed to measure $\theta_{13}$, the smallest mixing angle in the three-neutrino mixing framework, with unprecedented precision. The experiment consists of eight identically designed detectors placed underground at different baselines from three pairs of nuclear reactors in South China. Since Dec. 2011, the experiment has been running stably for more than 7 years, and has collected the largest reactor anti-neutrino sample to date. Daya Bay greatly improved the precision on $\theta_{13}$ and made an independent measurement of the effective mass splitting in the electron antineutrino disappearance channel. Daya Bay also performed a number of other precise measurements, such as a high-statistics determination of the absolute reactor antineutrino flux and spectrum evolution, as well as a search for sterile neutrino mixing, among others. The most recent neutrino oscillation results from Daya Bay are discussed in this article.

European Physical Society Conference on High Energy Physics - EPS-HEP2019 -

10-17 July, 2019

Ghent, Belgium

\footnotetext{
* Speaker.

${ }^{\dagger}$ on behalf of Daya Bay collaboration
} 


\section{The Daya Bay Neutrino Experiment}

The Daya Bay Reactor Neutrino Experiment was designed to measure $\theta_{13}$, the smallest mixing angle in the three-neutrino mixing framework, with unprecedented precision [1]. The experiment profits from a rare constellation of a nuclear power station complex situated near Hong Kong and adjacent mountains. The reactors serve as the neutrinos source while the mountains provide a sufficient overburden suppressing cosmic muons - the strongest background source.

At the time of the measurement the facility consisted of six pressurized water reactors (PWRs). The electron antineutrinos are emitted in beta-decay of fission fragments released in the chain reaction. The antineutrino flux and the energy spectrum is determined by the total thermal power of the reactor, the fraction of each fissile isotope in the fuel, the fission rate of each isotope and the energy spectrum of neutrinos from each isotope. All the reactors have the same thermal power 2.9 $\mathrm{GW}_{t h}$ each and all together produced roughly $3.5 \times 10^{21} \tilde{v}_{e} / \mathrm{s}$ with energies up to $8 \mathrm{MeV}$ making it one of the most intense $\tilde{v}_{e}$ sources on Earth.

Two antineutrino detectors installed in each underground experimental hall near to the reactors (Hall 1 and Hall 2) measured the $\tilde{v}_{e}$ flux emitted by the reactors, while four detectors in the far experimental hall (Hall 3) measured a deficit in the $\tilde{v}_{e}$ flux due to oscillation in the location where the neutrino oscillation effect is expected to be the strongest. Such configuration allows to suppress reactor related uncertainty in the measured neutrino flux. The disappearance signal is most pronounced at the first oscillation minimum. Based on existing accelerator and atmospheric neutrino oscillation measurements this corresponded to a distance $L_{f} \approx 1.6 \mathrm{~km}$ for reactor $\tilde{v}_{e}$ with a mean energy of $4 \mathrm{MeV}$.

Each detector contains 20 tons of Gd-loaded liquid scintillator (target) and 20 tons of normal liquid scintillator (gamma catcher) separated by acrylic vessels. The outermost layer of the detector contains mineral oil (shielding) inside of stainless steel vessel equipped with 192 8-inch PMTs. The detectors are submerged in a water pools instrumented as Cherenkov detectors and covered with 4-layers of Resistive Plate Chamber detectors for cosmic muon tagging.

\section{Results}

\subsection{Oscillation analysis based on n-Gd [3]}

The presented results are from analysis of data collected in the Daya Bay experiment with 6 detectors in 217 days (Dec/2011 - Jul/2012), with 8 detectors in 1524 days (Oct/2012 - Dec/2016) and with 7 detectors in 217 days (Jan/2017 - Aug/2017). During 1958 days of operation, the Daya Bay experiment collected more than 3.5 millions inverse beta decays in the near halls and more than 0.5 million IBD have been detected in the far hall. The daily rate is $\sim 2500$ IBD events in the near halls and $\sim 300$ IBD in the far hall.

The distortion of the energy spectrum at the far hall relative to near halls was consistent with oscillation, and allowed measurement of $\left|\Delta m_{e e}^{2}\right|$. The parameters of the three-flavor model in best 

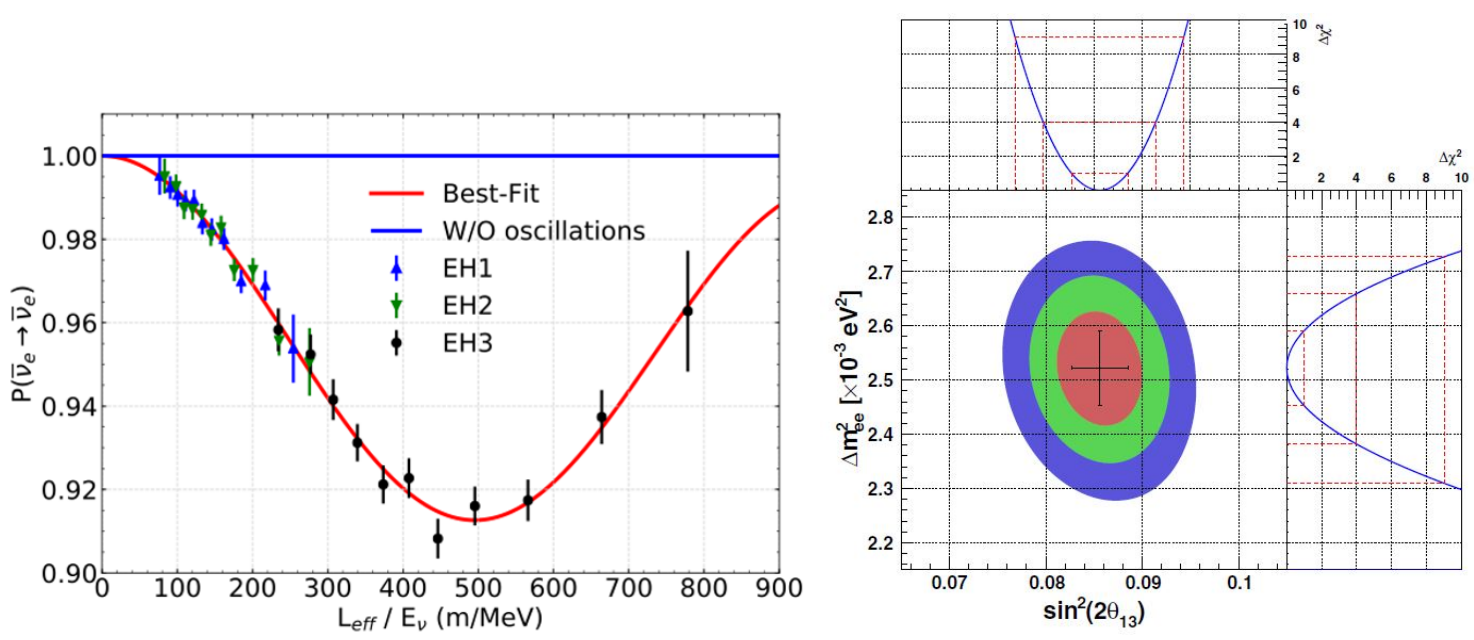

Figure 1: Oscillation survival probability versus antineutrino proper time - left. The $68.3 \%, 95.5 \%$ and 99.7\% C.L. allowed regions for $\sin ^{2} 2 \theta_{13}$ and $\left|\Delta m_{e e}^{2}\right|-$ right.

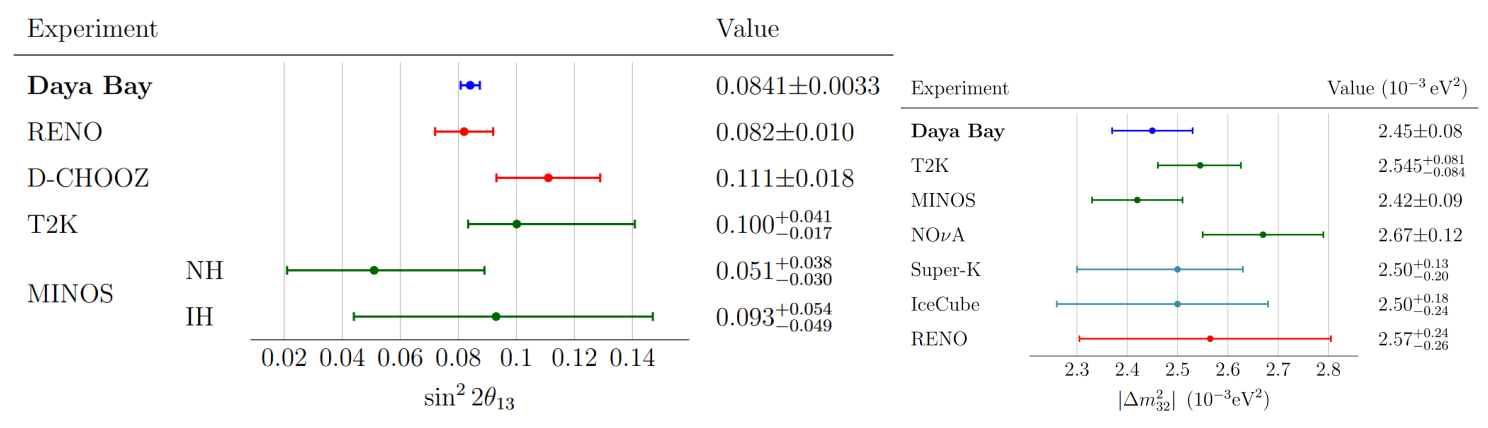

Figure 2: Comparison of $\sin ^{2} 2 \theta_{13}$ provided by RENO [4], D-CHOOZ [5], T2K [6] and MINOS [7], and $\left|\Delta m_{32}^{2}\right|$ values provided by RENO [4], T2K [6], MINOS [8], NOvA [9], Super-K [10] and IceCube [11].

agreement with the observed rate and energy spectra were

$$
\begin{aligned}
\sin ^{2} 2 \theta_{13} & =0.0856 \pm 0.0029 \\
\left|\Delta m_{e e}^{2}\right| & =\left[2.522_{-0.070}^{+0.068}\right] \times 10^{-3} \mathrm{eV}^{2} \\
\Delta m_{32}^{2}(N H) & =+\left[2.471_{-0.070}^{+0.068}\right] \times 10^{-3} \mathrm{eV}^{2} \\
\Delta m_{32}^{2}(I H) & =-\left[2.575_{-0.070}^{+0.068}\right] \times 10^{-3} \mathrm{eV}^{2}
\end{aligned}
$$

The $\Delta m_{32}^{2}$ values were obtained under the assumptions of normal (NH) and inverted (IH) mass ordering.

Figure 1 - left, shows the observed electron survival probability as a function of effective baseline $L_{\text {eff }}$ divided by the average antineutrino energy $\left\langle E_{v}\right\rangle$. Almost one full oscillation disappearance and reappearance cycle was sampled, given the range of $L / E_{v}$ values which were measured.

The confidence intervals for $\Delta m_{e e}^{2}$ versus $\sin ^{2} 2 \theta_{13}$ are shown in Figure 1 - right. The $1 \sigma, 2 \sigma$, and $3 \sigma$ 2-D confidence intervals are estimated using $\Delta \chi^{2}$ values of 2.30 (red), 6.18 (green), and 11.83 (blue) relative to the best fit. The upper panel provides the $1-\mathrm{D} \Delta \chi^{2}$ for $\sin ^{2} 2 \theta_{13}$ obtained by profiling $\left|\Delta m_{e e}^{2}\right|$ (blue line), and dash lines mark the corresponding $1 \sigma, 2 \sigma$, and $3 \sigma$ intervals. The 

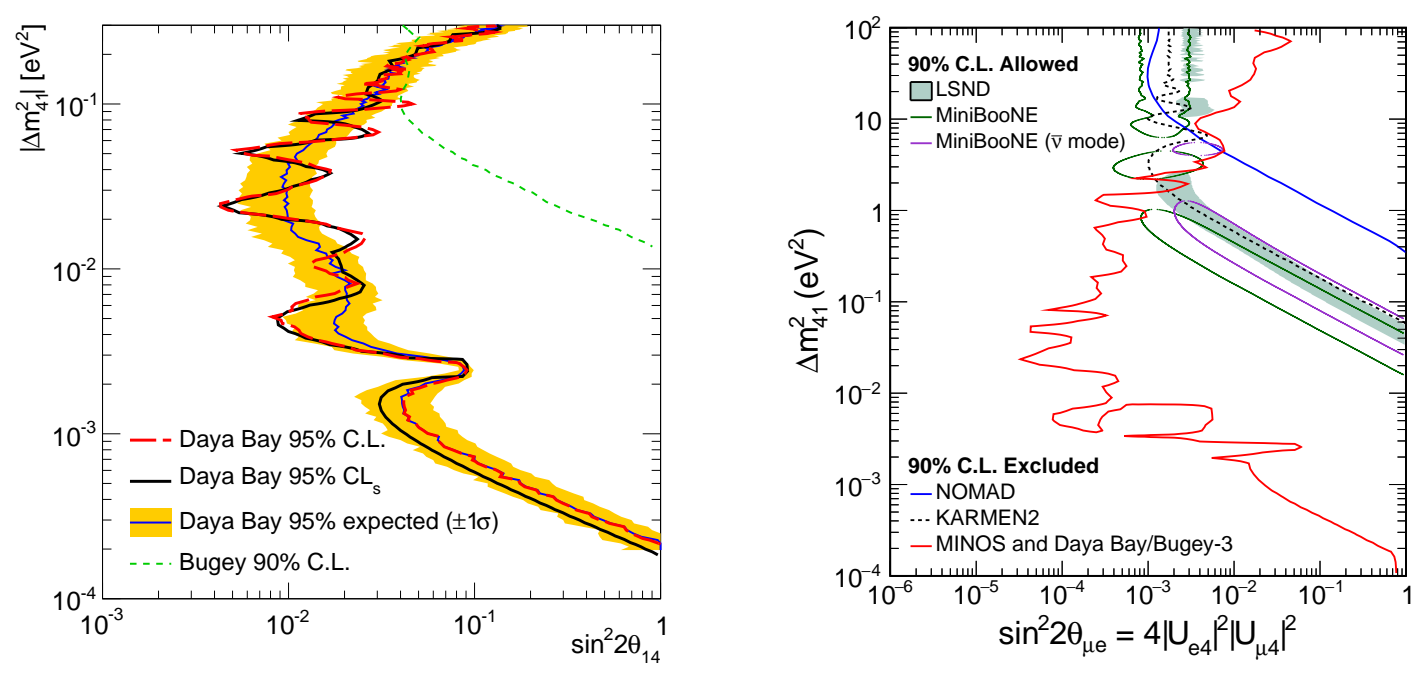

Figure 3: Constraints for sterile light neutrino provided by Daya Bay [13] - left, and combined analysis of data from MINOS and Daya Bay/Bugey-3 [14] - right.

right panel is the same, but for $\left|\Delta m_{e e}^{2}\right|$, with $\sin ^{2} 2 \theta_{13}$ profiled. The point marks the best estimates, and the error bars display their 1-D $1 \sigma$ confidence intervals.

The Daya Bay results are compatible with $\sin ^{2} 2 \theta_{13}$ results provided by other experiments: RENO [4], D-CHOOZ [5], T2K [6] and MINOS [7], and $\left|\Delta m_{32}^{2}\right|$ values provided by RENO [4], T2K [6], MINOS [8], NOvA [9], Super-K [10] and IceCube [11]. While acuracy of determination of $\left|\Delta m_{32}^{2}\right|$ is comparable with T2K and MINOS, determination of $\sin ^{2} 2 \theta_{13}$ is more then twice more acurate then other results.

\subsection{Oscillation analysis based on n-H [12]}

Alternative data analysis of data taken in 621 days, based on the events in which the neutron from IBD is captured on hydrogen results with

$$
\sin ^{2} 2 \theta_{13}=0.071 \pm 0.011
$$

Combination of the $\mathrm{n}-\mathrm{H}$ and $\mathrm{n}-\mathrm{Gd}$ results from 1230 days data gives $8 \%$ improvement in precision

$$
\sin ^{2} 2 \theta_{13}=0.082 \pm 0.004
$$

\subsection{Search for Light Sterile Neutrino}

The large statistics collected with full configuration of eight detectors in the Daya Bay experiment allowed new precise analysis with aim to search for a light sterile neutrino [13]. A relative comparison of the rate and energy spectrum of reactor antineutrinos in the three experimental halls yields no evidence of sterile neutrino mixing in the $2 \times 10^{-4}<\left|\Delta m_{41}^{2}\right|<0.3 \mathrm{eV}^{2}$ mass range. The resulting limits on $\sin ^{2} 2 \theta_{14}$ shown on the Figure 3 - left, constitute the most stringent constraints to date in the $\left|\Delta m_{41}^{2}\right|<0.2 \mathrm{eV}^{2}$ region. 
Searches for a light sterile neutrino have been independently performed by the MINOS and the Daya Bay experiments using the muon (anti)neutrino and electron antineutrino disappearance channels, respectively. Results from both experiments are combined with those from the Bugey-3 reactor neutrino experiment to constrain oscillations into light sterile neutrinos [14]. The three experiments are sensitive to complementary regions of parameter space, enabling the combined analysis to probe regions allowed by the LSND and MiniBooNE experiments in a minimally extended four-neutrino flavor framework. Stringent limits on $\sin ^{2} 2 \theta_{\mu e}$ are set over six orders of magnitude in the sterile mass-squared splitting $\Delta m_{41}^{2}$. The sterile-neutrino mixing phase space allowed by the LSND and MiniBooNE experiments is excluded for $\Delta m_{41}^{2}<0.8 \mathrm{eV}^{2}$ at $95 \% \mathrm{CLs}$, see the Figure 3 - right.

\section{References}

[1] An F. P. et al. (Daya Bay Collaboration) Phys. Rev. Lett. 2012. V.108. P.171803.

[2] Cowan C. L., Reines F., Harrison F. B., Kruse H. W., McGuire A. D. Science 1956. V.124. P.103.

[3] Adey D. et al. (Daya Bay Collaboration) Phys.Rev. Lett. 121, 241805 (2018).

[4] Choi J. H. et al. (RENO Collaboration) Phys. Rev. Lett. 2016. V.116. P.211801 (arXiv:1511.05849 [hep-ex])

[5] Ishitsuka M. (Double Chooz Collaboration) 51st Rencontres de Moriond, Electroweak Interactions and United Theories. 2016.

[6] Iwamoto K. (T2K Collaboration) 38th Int. Conf. on High Energy Physics. 2016.

[7] Adamson P. et al. (MINOS Collaboration) Phys. Rev. Lett. 2013. V.110. P.171801.

[8] Evans J. (MINOS and MINOS+ Collaborations) 27th Int. Conf. on Neutrino Physics and Astrophysics. 2016.

[9] Vahle P. (NOvA Collaboration) 27th Int. Conf. on Neutrino Physics and Astrophysics. 2016.

[10] Moriyama S. (Super-Kamiokande Collaboration) 27th Int. Conf. on Neutrino Physics and Astrophysics. 2016

[11] Koskinen J. (Ice Cube Collaboration) 27th Int. Conf. on Neutrino Physics and Astrophysics. 2016.

[12] An F. P. et al. (Daya Bay Collaboration) Phys. Rev. D 2016. V.93. P.072011.

[13] An F. P. et al. (Daya Bay Collaboration) Phys. Rev. Lett. 2016. V.117 no.15. P.151802.

[14] An F. P. et al. (Daya Bay Collaboration, MINOS Collaboration) Phys. Rev. Lett. 2016. V.117. no.15. P.151801.

Addendum: Phys. Rev. Lett. 2016. V.117. no.20 P.209901. 